\title{
Magnetic Properties of $\mathrm{La}_{0.8} \mathrm{~K}_{0.2} \mathrm{MnO}_{3}$ Nanoparticles
}

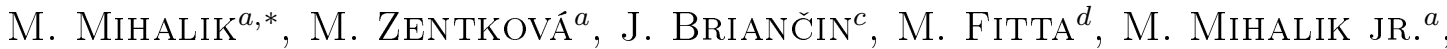 \\ J. LAZÚROVÁ ${ }^{a}$, M. VAVRA ${ }^{a, b}$
}

${ }^{a}$ Institute of Experimental Physics SAS, Watsonova 47, 04001 Košice, Slovakia

${ }^{b}$ Institute of Chemistry, Faculty of Science, P.J. Šafarik University, Moyzesova 11, 04154 Košice, Slovakia

${ }^{c}$ Institute of Geotechnics SAS, Watsonova 45, 04001 Košice, Slovakia

${ }^{d}$ Institute of Nuclear Physics, Polish Academy of Sciences, Radzikowskiego 152, Kraków, Poland

Magnetic properties of $\mathrm{La}_{0.8} \mathrm{~K}_{0.2} \mathrm{MnO}_{3}$ have been studied on nanoparticles prepared by glycine-nitrate method. Crystal structure and particles size were modified by heat treatment. Crystal structure changes from orthorhombic (space group $\mathrm{Pbma}$ ) to rhombohedral (space group $R$ - $3 \mathrm{c}$ ) after annealing at $600{ }^{\circ} \mathrm{C} / 2$ hours. The average size of particle varies with annealing from about $30 \mathrm{~nm}$ to $135 \mathrm{~nm}$. The Curie temperature $T_{C}$ and the saturated magnetization $\mu_{s}$ increase with annealing. The exchange bias effect was observed on samples with particles size smaller than $60 \mathrm{~nm}$.

DOI: $10.12693 /$ APhysPolA.126.312

PACS: 75.50.-y, 75.47.Lx, 75.30.-m, 75.30Kz

The mixed-valence manganese oxides of the general formula $\mathrm{La}_{1-x} \mathrm{~A}_{x} \mathrm{MnO}_{3}$ ( $\mathrm{A}$ is a divalent ion like $\mathrm{Ca}, \mathrm{Sr}$, $\mathrm{Ba}$ and $\mathrm{Pb}$ ) are a subject of interest due to a desire to understand and exploit the large negative magnetoresistance and magnetocaloric effects [1]. Among manganites, the manganites doped with univalent metals, such as $\mathrm{Ag}, \mathrm{K}$, and $\mathrm{Na}$ are of greatest interest, because their physical properties are very sensitive to magnetic field at room temperature. Group of $\mathrm{La}_{1-x} \mathrm{~K}_{x} \mathrm{MnO}_{3}$ manganites provides a series of new oxides to study magnetocaloric effect [2, 3] and insulator-to-metal transition [4] at room temperature. In our paper we study the effect of annealing on magnetic properties of $\mathrm{La}_{0.8} \mathrm{~K}_{0.2} \mathrm{MnO}_{3}$ nanoparticles.

The preparation of nanoparticles followed the glycinenitrate method, where glycine was used as a fuel and nitrates as oxidants [5]. The as prepared samples were annealed for 2 hours in air atmosphere at $300^{\circ} \mathrm{C}, 600^{\circ} \mathrm{C}$ and $900^{\circ} \mathrm{C}$.

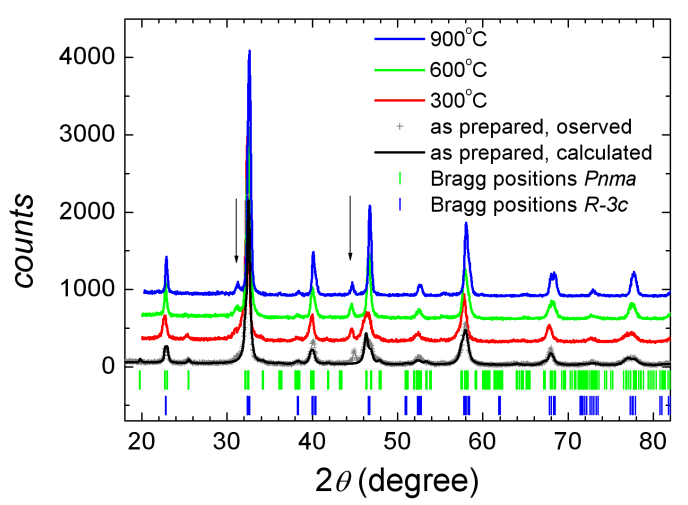

Fig. 1. X-ray powder diffraction patterns for all samples; arrows point to the contribution from sample holder.

*corresponding author; e-mail: mihalik@saske.sk

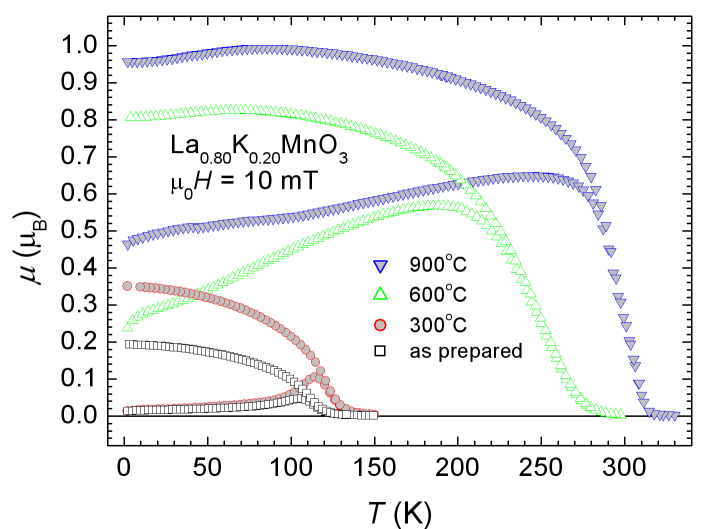

Fig. 2. Magnetization measurements in $\mathrm{ZFC}$ and FC regimes for all samples.

The X-ray powder diffraction (XRD) measurements have been carried out on the X'Pert PRO diffractometer with $\mathrm{Cu}-\mathrm{K}_{\alpha}$ radiation $\left(\lambda_{1}=1.54056 \AA, \lambda_{2}=1.54440 \AA\right)$ and the XRD patterns were evaluated with the FullProf program based on the Rietveld method [6]. The as prepared sample and sample annealed at $300^{\circ} \mathrm{C}$ adopt orthorhombic crystal structure (space group Pnma) with lattice parameters $a=0.5570(5) \mathrm{nm} ; b=0.7773(9) \mathrm{nm}$; $c=0.5525(1) \mathrm{nm}$. The crystal structure changes to rhombohedral (space group $R-3 c$ ) with annealing, $a=$ $0.5512(6) \mathrm{nm} ; c=1.3385(2) \mathrm{nm}$ for the sample annealed at $900{ }^{\circ} \mathrm{C} . \mathrm{MnO}_{6}$ - the building blocks of the crystal structures are distorted and tilted. The average size of nanoparticles depends on annealing and varies between $30 \mathrm{~nm}$ and $135 \mathrm{~nm}$. The morphology of nanoparticles and their size distribution was studied on powders by scanning electron microscope (SEM) MIRA3 TESCAN. Magnetization and AC susceptibility measurements were performed by a SQUID (MPMS XL-5) or in a VSM (PPMS) magnetometer in the temperature range from $1.8 \mathrm{~K}$ to $380 \mathrm{~K}$ and in magnetic field up to $9 \mathrm{~T}$.

Hysteretic behaviour (see Fig. 2) between ZFC and FC magnetization is a characteristic feature of the system. 


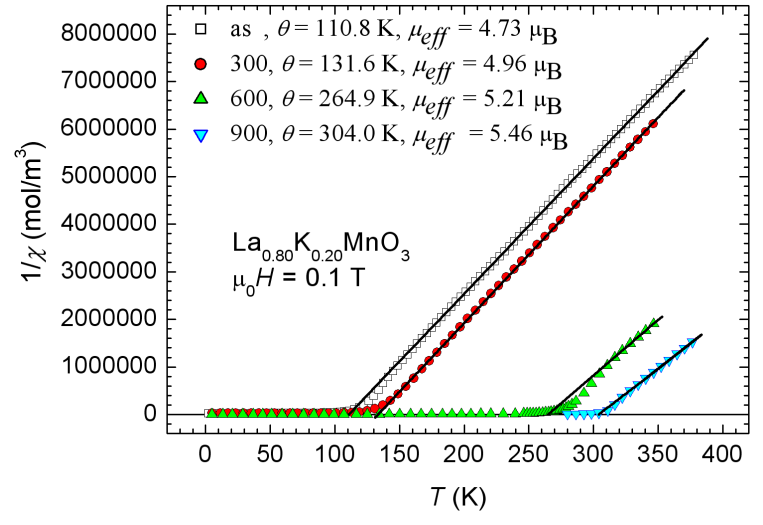

Fig. 3. The inverse susceptibility for all samples, lines represent fits to the Curie-Weiss law.

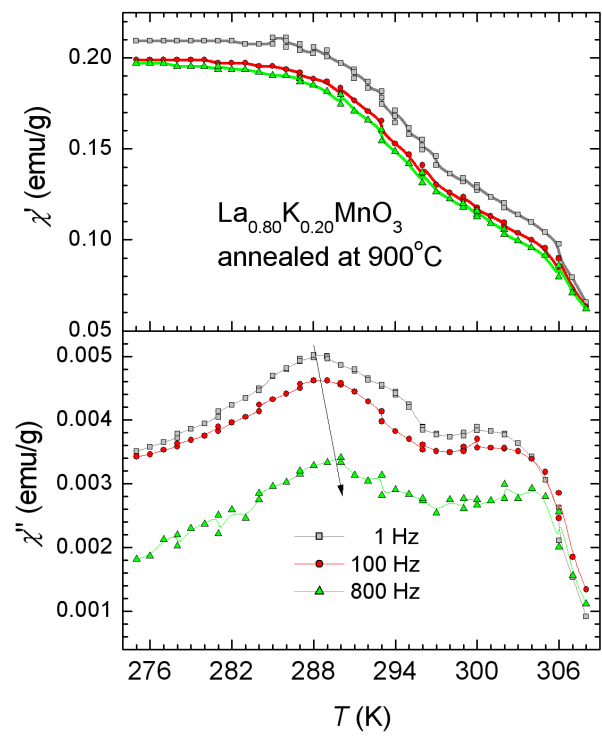

Fig. 4. In phase and out of phase AC susceptibility for the sample annealed at $900{ }^{\circ} \mathrm{C}$.

The increase of ferromagnetic interactions with annealing is evident from all measurements. The Curie temperature increases from $T_{C}=112.0 \mathrm{~K}$ to $295.0 \mathrm{~K}$ after annealing. The magnetic susceptibility obeys the CurieWeiss law (Fig. 3) for all samples. The paramagnetic Curie temperature $\theta$ and the effective magnetic moment $\mu_{\text {eff }}$ increase with annealing, indicating the increase of ferromagnetic interactions.

The magnetic phase transition is accompanied with an anomaly in both the real part $\chi^{\prime}$, and the imaginary part $\chi^{\prime \prime}$ of AC susceptibility, which are frequency dependent in the case of sample annealed at $900{ }^{\circ} \mathrm{C}$ (Fig. 4). In this case two maxima are seen in the susceptibility at the magnetic phase transition (Fig. 4) at $T_{C 1}=288 \mathrm{~K}$ and $T_{C 2}=302 \mathrm{~K}$, indicating magnetic inhomogenities in the material. Double transition was observed also in magnetization measurements.

The increase of ferromagnetic correlations in material is seen also from magnetic hysteresis loops measurements.
The saturated magnetization $\mu_{s}$ increases with annealing from $1.54 \mu_{B}$ for sample annealed at $30{ }^{\circ} \mathrm{C}$ to $3.29 \mu_{B}$ for sample annealed at $900^{\circ} \mathrm{C}$. The exchange bias effect (EB) was observed on as prepared sample and sample annealed at $300{ }^{\circ} \mathrm{C}$ (Fig. 5). In these cases the average particle size is less than $50 \mathrm{~nm}$ and core shell model can be applied to explain such behaviour. Cooling down in magnetic field $\mu_{0} H_{c f}=1 \mathrm{~T}$ gives rise to displacement of the magnetic hysteresis loop, which is the typical manifestation of the EB effect. The loop is pinned on vertex in the region of negative magnetization, it is tilted and shifted in horizontal and vertical direction. The horizontal shift of the loop is usually expressed by the exchange bias field $\mu_{0} H_{E}=\mu_{0}\left(H_{c+}-H_{c-}\right) / 2=1165 \mathrm{mT} ; H_{c+}$ and $H_{c-}$ are the coercive fields on positive and negative axis, respectively. The vertical shift is expressed by the remnant asymmetry $\mu_{E}=\left(\mu_{r+}-\mu_{r-}\right) / 2=0.517 \mu_{B} ; \mu_{r+}$ and $\mu_{r-}$ are the remnant magnetizations on the positive or the negative axis, respectively.

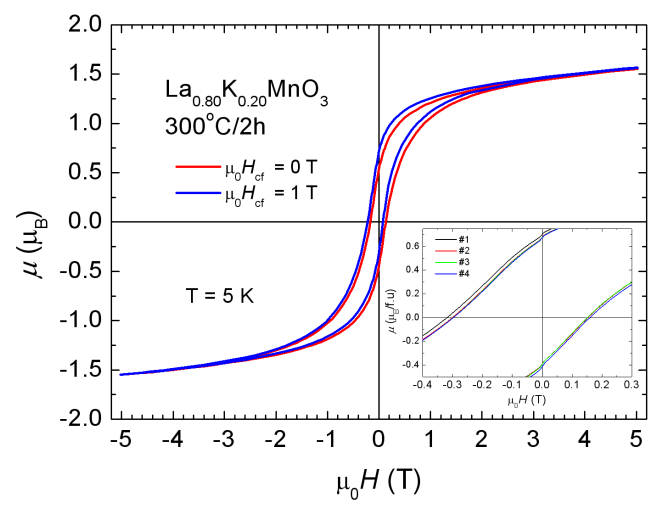

Fig. 5. Exchange bias phenomenon for the sample annealed at $300{ }^{\circ} \mathrm{C}$. The insert shows the training effect after 4 cycles.

This work was supported by the projects VEGA2/0178/13, APVV-0132-11 and ERDF EU under the contract No. ITMS26220120005.

\section{References}

[1] J.M.D. Coey, T. Venkatesan, A.J. Millis, J.R. Cooper, P.C. Riedi, P.B. Littlewood, D.M. Edwards, J.Z. Sun, J. Inoue, Philosophical Transactions: Math., Phys. and Eng. Sci. 365, 1519 (1998).

[2] A.M. Aliev, A.G. Gamzatov, A.B. Batdalov, A.S. Mankevich, I.E. Korsakov, Physica B 406, 885 (2011).

[3] I.K. Kamilov, A.G. Gamzatov, A.B. Batdalov, A.S. Mankevich, I.E. Korsakov, Phys. Solid State 52, 789 (2010).

[4] C. Shivakumara, M.B. Bellakki, Bull. Mater. Sci. 32, 443 (2009).

[5] D. Markovic, V. Kusigerski, M. Tadic, J. Blanusa, M.V. Antisari, V. Spasojevic, Scr. Mater. 59, 35 (2008).

[6] M.H. Rietveld, J. Appl. Cryst. 2, 65 (1969). 